\title{
Long Time Deviations from the Exponential Decay Law: Possible Observational Effects
}

\author{
K. Urbanowski, J. Piskorski \\ University of Zielona Góra, Institute of Physics, \\ ul. Prof. Z. Szafrana 4a, 65-516 Zielona Góra, Poland \\ e-mail: \{K.Urbanowski/J.Piskorski\}@proton.if.uz.zgora.pl
}

(Received: 23 March 2010; accepted: 5 July 2010; published online: 1 September 2010)

\begin{abstract}
An effect generated by the nonexponential behavior of the survival amplitude of an unstable state in the long time region is considered. We find that the instantaneous energy of the unstable state for a large class of models of unstable states tends to the minimal energy of the system $\mathcal{E}_{\min }$ as $t \rightarrow \infty$ which is much smaller than the energy of this state for $t$ of the order of the lifetime of the considered state. Analyzing the transition time region between the exponential and non-exponential form of the survival amplitude, we find that the instantaneous energy of the considered unstable state can take large values, much larger than the energy of this state for $t$ from the exponential time region. Taking into account results obtained for a considered model, it is hypothesized that this purely quantum mechanical effect may be responsible for the properties of broad resonances such as $\sigma$ meson as well as having astrophysical and cosmological consequences
\end{abstract}

Key words: decay law, non exponential decay

\section{INTRODUCTION}

Searching for the properties of unstable states $|\varphi\rangle \in \mathcal{H}$ (where $\mathcal{H}$ is the Hilbert space of states of the considered system) one analyzes their decay law. The decay law, $\mathcal{P}_{\varphi}(t)$ of an unstable state $|\varphi\rangle$ decaying in vacuum is defined as follows

$$
\mathcal{P}_{\varphi}(t)=|a(t)|^{2}
$$

where $a(t)$ is the probability amplitude of finding the system at time $t$ in the initial state $|\varphi\rangle$ prepared at time $t_{0}=0$,

$$
a(t)=\langle\varphi \mid \varphi(t)\rangle \text {, }
$$

and $|\varphi(t)\rangle$ is the solution of the Schrödinger equation for the initial condition $|\varphi(0)\rangle=|\varphi\rangle$. From basic principles of the quantum theory it is known that the amplitude $a(t)$, and thus the decay law $\mathcal{P}_{\varphi}(t)$ of the unstable state $|\varphi\rangle$, are completely determined by the density of the energy distribution $\omega(\mathcal{E})$ for the system in this state $[1,2]$

$$
a(t)=\int_{\text {Spec. }(H)} \omega(\mathcal{E}) e^{-\frac{i}{\hbar} \mathcal{E} t} d \mathcal{E},
$$

where $\omega(\mathcal{E}) \geq 0$ and $a(0)=1$.
In [3], assuming that the spectrum of $H$ must be bound from below, (Spec. $(H)>-\infty)$, and using the Paley-Wiener Theorem [4], it was proved that in the case of unstable states there must be $|a(t)| \geq A \exp \left[-b t^{q}\right]$ for $|t| \rightarrow \infty$ (where $A>0, b>0$ and $0<q<1)$.

The problem of how to detect possible deviations from the exponential form of $\mathcal{P}_{\varphi}(t)$ at the long time region has been attracting the attention of physicists since the first theoretical predictions of such an effect. Many tests of the decay law performed some time ago did not indicate any deviations from the exponential form of $\mathcal{P}_{\varphi}(t)$ at the long time region. Nevertheless, conditions leading to the nonexponential behavior of the amplitude $a(t)$ at long times were studied theoretically. Conclusions following from these studies were applied successfully in an experiment described in the Rothe paper [5], where the experimental evidence of deviations from the exponential decay law at long times was reported. This result gives rise to another problem which now becomes important: if (and how) deviations from the exponential decay law at long times affect the energy of the unstable state and its decay rate at this time region.

Note that in fact the amplitude $a(t)$ contains information about the decay law $\mathcal{P}_{\varphi}(t)$ of the state $|\varphi\rangle$, that is about 
the decay rate $\gamma_{\varphi}^{0}$ of this state, as well as the energy $\mathcal{E}_{\varphi}^{0}$ of the system in this state. This information can be extracted from $a(t)$. Indeed, if $|\varphi\rangle$ is an unstable (a quasi-stationary) state, then there is

$$
\mathcal{E}_{\varphi}^{0}-\frac{i}{2} \gamma_{\varphi}^{0} \equiv i \hbar \frac{\partial a_{0}(t)}{\partial(t)} \frac{1}{a_{0}(t)},
$$

for $t \sim \tau_{\varphi}$, where

$$
a_{0}(t)=\exp \left[-\frac{i}{\hbar}\left(\mathcal{E}_{\varphi}^{0}-\frac{i}{2} \gamma_{\varphi}^{0}\right) t\right] \simeq a(t)
$$

for $t \sim \tau_{\varphi}, \tau_{\varphi}=\frac{\hbar}{\gamma_{\varphi}^{0}}$ and $\gamma_{\varphi}^{0}$ is the decay rate of $|\varphi\rangle$.

Taking the above into account one can define the "effective Hamiltonian", $h_{\varphi}$, for the one-dimensional subspace of states $\mathcal{H}_{\|}$spanned by the normalized vector $|\varphi\rangle$ as follows (see, eg. [6]),

$$
h_{\varphi} \stackrel{\operatorname{def}}{=} i \hbar \frac{\partial a(t)}{\partial t} \frac{1}{a(t)} .
$$

So let us assume that we know the amplitude $a(t)$. Then starting with this $a(t)$ and using the expression (5), one can calculate the effective Hamiltonian $h_{\varphi}(t)$ in a general case for every $t$. Thus, one finds the following expressions for the energy and the decay rate of the system in the state $|\varphi\rangle$ under consideration, to be more precise for the instantaneous energy and the instantaneous decay rate, (for details see: [7, 8]),

$$
\begin{gathered}
\mathcal{E}_{\varphi} \equiv \mathcal{E}_{\varphi}(t)=\Re\left(h_{\varphi}(t)\right), \\
\gamma_{\varphi} \equiv \gamma_{\varphi}(t)=-2 \mathfrak{I}\left(h_{\varphi}(t)\right),
\end{gathered}
$$

where $\mathfrak{R}(z)$ and $\mathfrak{I}(z)$ denote the real and imaginary parts of $z$, respectively.

The aim of this paper is to discuss the long time behaviour of $\mathcal{E}_{\varphi}(t)$ using $a(t)$ calculated for the given density $\omega(\mathcal{E})$.

\section{THE MODEL}

Let us assume that $\operatorname{Spec} .(H)=\left[\mathcal{E}_{\min }, \infty\right)$, (where $\left.\mathcal{E}_{\min }>-\infty\right)$, and let us choose $\omega(\mathcal{E})$ as follows

$$
\omega(\mathcal{E}) \equiv \omega_{B W}\left(\mathcal{E}, \mathcal{E}_{\min }\right)=\frac{N}{2 \pi} \Theta\left(\mathcal{E}-\mathcal{E}_{\min }\right) \frac{\gamma_{\varphi}^{0}}{\left(\mathcal{E}-\mathcal{E}_{\varphi}^{0}\right)^{2}+\left(\frac{\gamma_{\varphi}^{0}}{2}\right)^{2}},(8)
$$

where $N$ is a normalization constant and $\Theta(\mathcal{E})=\{1$ for $\mathcal{E} \geq 0$, and 0 for $\mathcal{E}<0\}$. An substitution of this $\omega_{B W}(\mathcal{E}$, $\mathcal{E}_{\min }$ ) into (3) leads to the result (see also [9])

$$
\begin{gathered}
a(t)=N e^{-\frac{i}{\hbar}\left(\mathcal{E}_{\varphi}^{0}-i \frac{\gamma_{\varphi}^{0}}{2}\right) t} \times \\
\times\left\{1-\frac{i}{2 \pi}\left[e^{\frac{\gamma_{\varphi}^{0} t}{\hbar}} E_{1}\left(-\frac{i}{\hbar}\left(\mathcal{E}_{\varphi}^{0}-\mathcal{E}_{\min }+\frac{i}{2} \gamma_{\varphi}^{0}\right) t\right)+\right.\right. \\
\left.\left.-E_{1}\left(-\frac{i}{\hbar}\left(\mathcal{E}_{\varphi}^{0}-\mathcal{E}_{\min }-\frac{i}{2} \gamma_{\varphi}^{0}\right) t\right)\right]\right\},
\end{gathered}
$$

where $E_{1}(x)$ denotes the integral-exponential function $[9,10]$. In general one has

$$
a(t) \equiv a_{\exp }(t)+a_{\mathrm{non}}(t)
$$

where

$$
a_{\exp }(t)=N \exp \left[-\frac{i}{\hbar}\left(\mathcal{E}_{\varphi}^{0}-i \frac{\gamma_{\varphi}^{0}}{2}\right) t\right]
$$

and

$$
a_{\mathrm{non}}(t)=a(t)-a_{\mathrm{exp}}(t)
$$

Making use of the asymptotic expansion of $E_{1}(x)$ [10] and (5) one finds

$$
\begin{gathered}
\left.\quad h_{\varphi}(t)\right|_{t \rightarrow \infty}=\left.i \hbar \frac{\partial a(t)}{\partial t} \frac{1}{a(t)}\right|_{t \rightarrow \infty} \simeq \\
\simeq \mathcal{E}_{\text {min }}-i \frac{\hbar}{t}-2 \frac{\mathcal{E}_{\varphi}^{0}-\mathcal{E}_{\text {min }}}{\left|h_{\varphi}^{0}-\mathcal{E}_{\text {min }}\right|^{2}}\left(\frac{\hbar}{t}\right)^{2}+\ldots
\end{gathered}
$$

for the considered case (8) of $\omega_{B W}\left(\mathcal{E}, \mathcal{E}_{\text {min }}\right.$ ), (for details see [7]). Here $h_{\varphi}^{0}=\mathcal{E}_{\varphi}^{0}-(i / 2) \gamma_{\varphi}^{0}$. From (11) it follows that

$$
\begin{gathered}
\Re\left(\left.h_{\phi}(t)\right|_{t \rightarrow \infty}\right) \stackrel{\text { def }}{=} \mathcal{E}_{\phi}^{\infty}(t) \simeq \\
\simeq \mathcal{E}_{\text {min }}-2 \frac{\mathcal{E}_{\phi}^{0}-\mathcal{E}_{\text {min }}}{\left|h_{\phi}^{0}-\mathcal{E}_{\text {min }}\right|^{2}}\left(\frac{\hbar}{t}\right)^{2} \underset{t \rightarrow \infty}{\rightarrow} \mathcal{E}_{\text {min }},
\end{gathered}
$$

where $\mathcal{E}_{\varphi}^{\infty}(t)=\left.\mathcal{E}_{\varphi}(t)\right|_{t \rightarrow \infty}$, and $\mathfrak{I}\left(\left.h_{\varphi}(t)\right|_{t \rightarrow \infty}\right) \simeq-\hbar / t$ for $t \rightarrow \infty$.

Relations (11), (12) become important for times $t>t_{a s}$, where $t_{a s}$ denotes the time $t$ at which contributions to $\left.|a(t)|_{t \rightarrow \infty}\right|^{2}$ from the exponential component and from the nonexponential component proportional to $1 / t^{2}$ are comparable, that is (see (10)),

$$
\left|a_{\text {exp }}(t)\right|^{2} \simeq\left|a_{\text {non }}(t)\right|^{2}
$$

for $t \rightarrow \infty$. 


\section{NUMERICAL CALCULATIONS}

Long time properties of the survival probability $|a(t)|^{2}$ and instantaneous energy $\mathcal{E}_{\varphi}(t)$ are relatively easy to find analytically for times $t \gg t_{a s}$ even in the general case as it was shown in the previous section and [8]. It is much more difficult to analyze these properties analytically in the transition time region where $t \sim t_{a s}$. It can be done numerically for given models.

The model considered in Sec. II and defined by the density $\omega_{B W}\left(\mathcal{E}, \mathcal{E}_{\text {min }}\right),(8)$, allows one to find numerically the decay curves and the instantaneous energy $\mathcal{E}_{\varphi}(t)$ as a function of time $t$. The results presented in this section have been obtained assuming for simplicity that the minimal energy $\mathcal{E}_{\text {min }}$ appearing in the formula (8) is equal to zero, $\mathcal{E}_{\text {min }}=0$. So, all numerical calculations were performed for the density $\tilde{\omega}_{B W}(\mathcal{E}) \equiv \omega_{B W}\left(\mathcal{E}, \mathcal{E}_{\text {min }}=0\right)$ for some chosen $\mathcal{E}_{\varphi}^{0} / \gamma_{\varphi}^{0}$. When performing calculations particular attention was paid to the form of the probability $|a(t)|^{2}$, i.e. of the decay curve, and of the instantaneous energy $\mathcal{E}_{\varphi}(t)$ for times $t$ belonging to the most interesting transition time-region between exponential and nonexponential parts of $|a(t)|^{2}$, where the property (13) takes place. Results are presented graphically below:

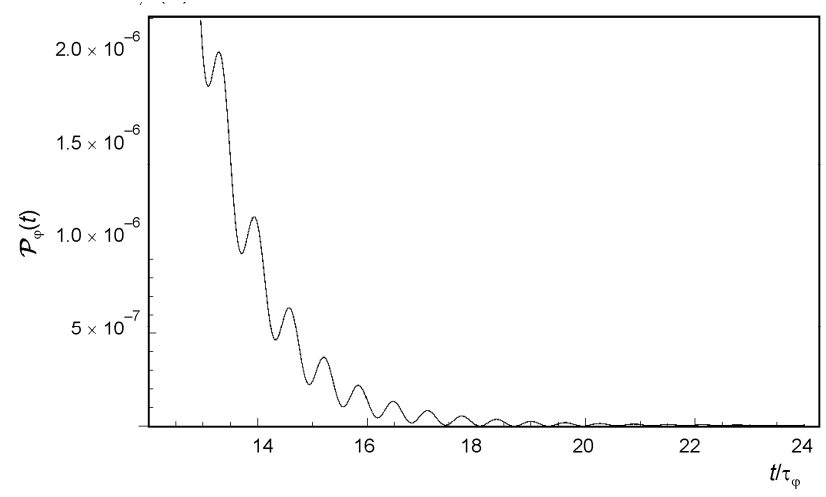

Fig. 1. Survival probability $\mathcal{P}_{\varphi}(t)=|a(t)|^{2}$ in the transition time region. The case $\mathcal{E}_{\varphi}^{0} / \gamma_{\varphi}^{0}=10$

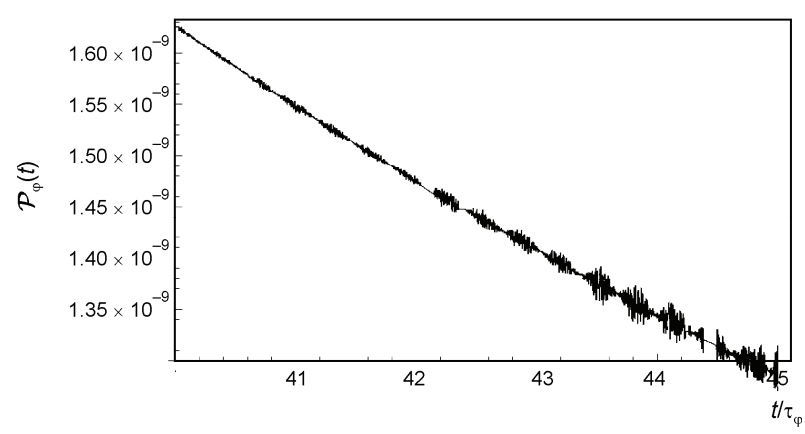

Fig. 2. Survival probability $\mathcal{P}_{\varphi}(t)=|a(t)|^{2}$ in the transition time region. The case $\mathcal{E}_{\varphi}^{0} / \gamma_{\varphi}^{0}=10$

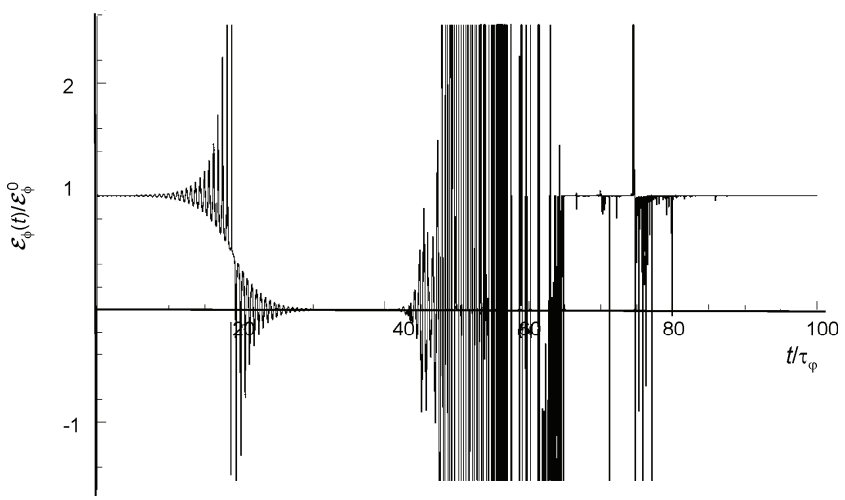

Fig. 3. Instantaneous energy $\mathcal{E}_{\varphi}(t)$ in the transition time region. The case $\mathcal{E}_{\varphi}^{0} / \gamma_{\varphi}^{0}=10$

\section{FINAL REMARKS}

Decay curves of shapes similar to those in Figs. (1), (2), are found in a large class of models (see [2, 11]). The characteristic feature of all these decay curves is the presence of sharp and frequent oscillations at the transition times region (see Figs. (1), (2)). This means that derivatives of the amplitude $a(t)$ may reach extremely large values for some times from the transition time region and the modulus of these derivatives is much larger than the modulus of $a(t)$, which is very small for these times. This explains why in this time region the real and imaginary parts of $h_{\varphi}(t) \equiv$ $\mathcal{E}_{\varphi}(t)-(i / 2) \gamma_{\varphi}(t)$, which can be expressed by the relation (5), i.e. by a large derivative of $a(t)$ divided by a very small $a(t)$, reach values much larger than the energy $\mathcal{E}_{\varphi}^{0}$ of the unstable state measured at times for which the decay curve has the exponential form. For the considered model we found that e.g. for $\mathcal{E}_{\varphi}^{0} / \gamma_{\varphi}^{0}=10$ and $5 \tau_{\varphi} \leq t \leq 60 \tau_{\varphi}$ the maximal value of the instantaneous energy equals $\mathcal{E}_{\varphi}(t)=$ $=89.2209 \mathcal{E}_{\varphi}^{0}$ and $\mathcal{E}_{\varphi}(t)$ reaches this value for $t \equiv t_{\mathrm{mx}}, 10=$ $=53.94 \tau_{\varphi}$ and then the survival probability $\mathcal{P}_{\varphi}(t)$ is of order $\mathcal{P}_{\varphi}\left(t_{m x}, 10\right) \sim 10^{-9}$.

The question is whether and where this effect can manifest itself. There are two possibilities to observe the above long time properties of unstable states: the first one is that one should analyze the properties of unstable states charactized by $t_{a s}$ which are not too long. The second one is finding a possibility to observe a suitably large number of events, i.e. unstable particles created by the same source.

The problem with understanding the properties of broad resonances in the scalar sector ( $\sigma$ meson problem [12]) discussed in $[13,14]$, where the hypothesis was formulated that this problem could be connected with properties of the decay amplitude in the transition time region, seems to be possible manifestations of this effect and this problem refers to the first possibility mentioned above. There is the 
problem with determining the mass of broad resonances. The measured range of a possible mass of $\sigma$ meson is very wide, 400-1200 MeV. Hence, one can not exclude the possibility that the masses of some $\sigma$ mesons are measured for times of the order of their lifetime, and some of them for times where their instantaneous energy $\mathcal{E}_{\sigma}(t)$ is much larger. This is exactly the case presented in Fig. (3). For broad mesons the ratio $\mathcal{E}_{\sigma}^{0} / \gamma_{\sigma}^{0}$ is relatively small and thus the time $t_{a s}$ when the above discussed effect occurs appears to be not too long.

Astrophysical and cosmological processes in which extremely huge numbers of unstable particles are created seem to be another possibility for the above discussed effect to become manifest. The probability $\mathcal{P}_{\varphi}(t)=|a(t)|^{2}$ that an unstable particle, say $\varphi$, survives up to time $t \sim t_{\text {as }}$ is extremely small. Let $\mathcal{P}_{\varphi}(t)$ be

$$
\left.\mathcal{P}_{\varphi}(t)\right|_{t \sim t_{a s}} \sim 10^{-k}
$$

where $k \gg 1$, then there is a chance to observe some of particles $\phi$ survived at $t \sim t_{a s}$ only if there is a source creating these particles in $\mathcal{N}_{\varphi}$ number so that

$$
\left.\mathcal{P}_{\varphi}(t)\right|_{t \sim t} \mathcal{N}_{\varphi} \gg 1
$$

So if a source exists that creates a flux containing

$$
\mathcal{N}_{f} \sim 10^{l}
$$

unstable particles and $l \gg k$ then the probability theory states that the number $N_{\text {surv }}$ unstable particles

$$
N_{\text {surv }}=\left.\mathcal{P}_{\varphi}(t)\right|_{t \sim t_{\text {as }}} \mathcal{N}_{\varphi} \sim 10^{l-k} \gg 0,
$$

has to survive up to time $t \sim t_{a s}$. Sources creating such numbers of unstable particles are known from cosmology and astrophysics. The Big Bang is the obvious example of such a source. Some other examples include processes taking place in galactic nuclei (galactic cores) and inside stars, etc.

Let us assume that we have an astrophysical source creating a sufficiently large number of unstable particles in a unit of time and emitting a flux of these particles and that this flux is constant or slowly varying in time. Let us consider a flux of neutrons. From (13) it follows that for the neutron $t_{a s}^{n} \sim\left(250 \tau_{n}-300 \tau_{n}\right)$, where $\tau_{n} \simeq 886$ [s]. If the energies of these neutrons are of order $30 \times 10^{17}[\mathrm{eV}]$ then during time $t \sim t_{a s}^{n}$ they can reach a distance $d_{n} \sim 25000$ [ly], that is the distance of about half of the Milky Way radius. If in a unit ot time a suitably large number of neutrons $\mathcal{N}_{n}$ of the energies mentioned is created by this source, then in the distance $d_{n}$ from the source a number of spherically symmetric space areas (halos) surrounding the source, where neutron instantaneous energies $\mathcal{E}_{n}(t)$ are much larger than their energy

$$
\mathcal{E}_{n}^{0}=\frac{m_{n}^{0} c^{2}}{\sqrt{1-\left(\frac{v_{n}}{c}\right)^{2}}}
$$

$\left(m_{n}^{0}\right.$ is the neutron rest mass and $v_{n}$ denotes its velocity) have to appear (see Fig. (4)). This conclusion obviously holds also for other unstable particles $\varphi_{\alpha}$ produced by this source.

Every kind of particles $\varphi_{\alpha}$ has its own halos located at distances $d_{k}^{\varphi_{a}} \sim v_{\varphi_{\alpha}} t_{a s}^{\varphi, k},(k=1,2, \ldots)$, from the source. Radiuses $d_{k}^{\varphi_{a}}$ of these halos are determined by the particles' velocities $v_{\varphi_{\alpha}}$ and by times $t_{a s}^{\varphi, k}$ when instantaneous energies $\mathcal{E}_{\varphi_{\alpha}}(t)$ have local maxima.

Unstable particles $\varphi_{\alpha}$ forming these halos and having instantaneous energies

$$
\mathcal{E}_{\varphi_{\alpha}}(t) \gg \mathcal{E}_{\varphi_{\alpha}}^{0}=m_{\varphi_{\alpha}}^{0} c^{2}
$$

have to interact gravitationally with objects outside these halos as particles of masses

$$
m_{\varphi_{\alpha}}(t)=\frac{1}{c^{2}} \mathcal{E}_{\varphi_{\alpha}}(t) \gg m_{\varphi_{\alpha}}^{0} .
$$

The possible observable effects depend on the astrophysical source of these particles considered.

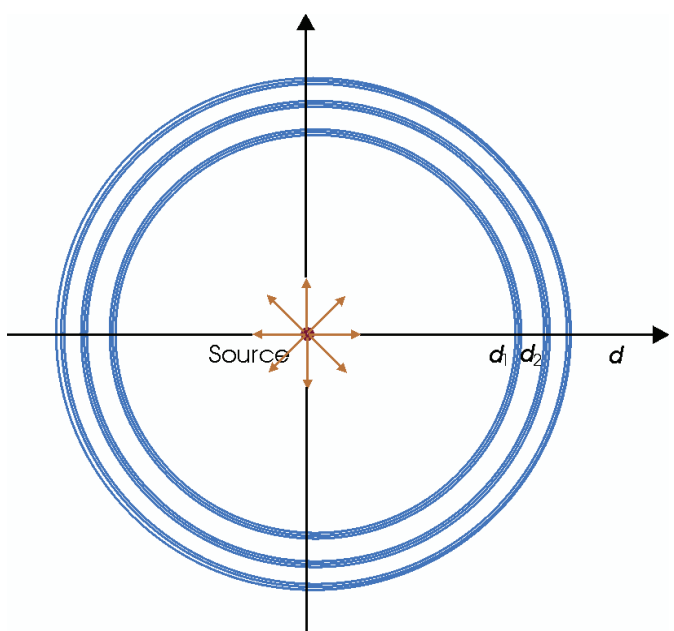

Fig. 4. Halos surrounding a source of unstable particles

If the halos are formed by unstable particles emitted as a result of internal star processes, then in the case of very young stars cosmic dust and gases should be attracted by these halos as a result of gravity attraction. So, the halos should be places where the dust and gases condensate. Thus in the case of very young stars one may consider the 
halos as the places where planets are born. On the other hand, in the case of much older stars the presence of halos should manifest itself in tiny changes of velocities and accelerations of object moving in the considered planetary star system relative to those calculated without taking into account of the halos presence.

If the halos are formed by unstable particles emitted by a galaxy core and these particles are such that the ratio $\mathcal{E}_{\varphi}(t) / \mathcal{E}_{\varphi}^{0}$ is suitably large inside the halos, then rotational velocities of stars rounding the galaxy center outside the halos should differ from those calculated without taking into account the halos. Thus the halos may affect the form of rotation curves of galaxies (we do not assume that the sole factor affecting the form of the rotation curves are these halos). Another possible effect is that the velocities of particles crossing these galactic halos should slightly vary in time due to gravitational interactions, i.e. they should gain some acceleration. This should cause charged particles to emit electromagnetic radiation when they cross the halo.

Note that the above mentioned effects seem to be possible to examine. All these effects are the simple consequence of the fact that the instantaneous energy $\mathcal{E}_{\varphi_{q}}(t)$ of unstable particles becomes large compared with $\mathcal{E}_{\varphi_{\alpha}}^{0}$ and for some times even extremely large. On the other hand, this property of $\mathcal{E}_{\varphi_{\alpha}}(t)$ results from the rigorous analysis of properties of the quantum mechanical survival probability $a(t)$ (see (2)) and from the assumption that the energy spectrum is bound from below.

\section{References}

[1] S. Krylov, V.A. Fock, Zh. Eksp. Teor. Fiz. 17, 93 (1947).

[2] L. Fonda, G.C. Ghirardii, A. Rimini, Rep. on Prog. in Phys. 41, 587 (1978).

[3] L.A. Khalfin, Zh. Eksp. Teor. Fiz. 33, 1371 (1957), [Sov. Phys. - JETP 6, 1053 (1958)].

[4] R.E.A.C. Paley, N. Wiener, Fourier transforms in the complex domain. American Mathematical Society, New York (1934).

[5] C. Rothe, S.I. Hintschich, A.P. Monkman, Phys. Rev. Lett. 96, 163601 (2006).

[6] K. Urbanowski, Phys. Rev. A 50, 2847 (1994).

[7] K. Urbanowski, Cent. Eur. J. Phys. 7 (2009), DOI:10.2478/s11534-009-0053-5.

[8] K. Urbanowski, Eur. Phys. J. D 54, 25 (2009).

[9] K.M. Sluis, E.A. Gislason, Phys. Rev. A 43, 4581 (1991).

[10] Handbook of Mathematical Functions, Natl. Bur. Stand. Appl. Math. Ser. No 55, eds. M. Abramowitz, I.A. Stegun (U.S. GPO, Washington, D.C., 1964).

[11] N.G. Kelkar, M. Nowakowski, K.P. Khemchadani, Phys. Rev. C 70, 024601 (2004).

[12] C. Amsler at al., Phys. Lett. B 667, 1 (2008).

[13] M. Nowakowski, N.G. Kelkar, Nishiharima 2004, Penataquark - Proceedings of International Workshop on PENATAQUARK 04, Spring - 8, Hyogo, Japan, 23-24 July 2004, pp. 182-189; arXiv: hep-ph/0411317.

[14] M. Nowakowski, N.G. Kelkar, AIP Conf. Proc. 1030, 250255 (2008); ArXiv:0807.5103.

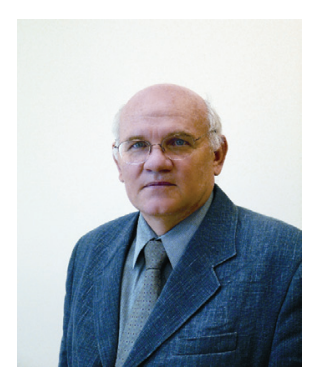

KrZYSZTOF UrbanowsKi. Education: Wrocław University - MSc (1973), PhD (1977); St-Petersburg State University - Dr hab. (1993). Activities: Theoretical physics - mathematical methods in physics, single and successive multiple measurements in quantum mechanics, early time and long time properties of quantum evolution, unstable states, CP and CPT symmetries in neutral meson complexes, evolution equations for neutral meson and multi-particle complexes.

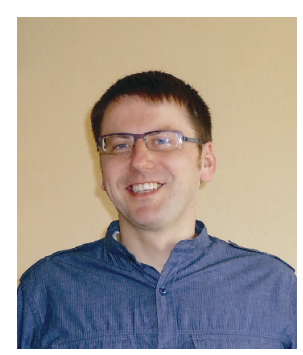

Dr. JarosŁaw Piskorski works at the Institute of Physics of the University of Zielona Góra, Poland. His main areas of interest include the application of the methods of statistical physics and nonlinear dynamics to heart rate variability and elementary particle physics (mainly discrete symmetries and their violation). Together with Prof. Przemyslaw Guzik, he co-discovered the Heart Rate Asymmetry phenomenon and cocreated the monotonic runs method in mortality prediction. 